\title{
Radio follow-up observations of stellar tidal disruption flares: Constraints on off-axis jets
}

\author{
S. van Velzen ${ }^{1, a}$, D.A. Frail ${ }^{2}$, E. Körding ${ }^{1}$, and H. Falcke ${ }^{1,3,4}$ \\ ${ }_{1}^{1}$ IMAPP, Radboud University, PO Box 9010, 6500 GL Nijmegen, The Netherlands \\ 2 National Radio Astronomy Observatory, Socorro, NM, USA \\ ${ }^{3}$ ASTRON, Dwingeloo, The Netherlands \\ ${ }^{4}$ Max-Planck-Institut für Radioastronomie Bonn, Germany
}

\begin{abstract}
Observations of active galactic nuclei (AGN) and X-ray binaries have shown that relativistic jets are ubiquitous when compact objects accrete. One could therefore anticipate the launch of a jet after a star is disrupted and accreted by a massive black hole. This birth of a relativistic jet may have been observed recently in two stellar tidal disruption flares (TDFs), which were discovered in gamma-rays by Swift. Yet no transient radio emission has been detected from the tens of TDF candidates that were discovered at optical to soft X-ray frequencies. Because the sample that was followed-up at radio frequencies is small, the non-detections can be explained by Doppler boosting, which reduces the jet flux for off-axis observers. Plus, the existing followup observation are mostly within $\sim 10$ months of the discovery, so the non-detections can also be due to a delay of the radio emission with respect to the time of disruption. To test the conjecture that all TDFs launch jets, we obtained $5 \mathrm{GHz}$ followup observations with the Jansky VLA of six known TDFs. To avoid missing delayed jet emission, our observations probe 1-8 years since the estimated time of disruption. None of the sources are detected, with very deep upper limits at the 10 micro Jansky level. These observations rule out the hypothesis that these TDFs launched jets similar to radio-loud quasars. We also constrain the possibility that the flares hosted a jet identical to Sw 1644+57.
\end{abstract}

\section{INTRODUCTION}

A star that passes too close to a massive black hole will be torn apart by tidal forces. The stellar debris that remains bound after this disruption returns to the black hole at a rate that initially can exceed the Eddington limit $\left(\dot{M}_{\text {Edd }}\right)$ by many orders of magnitude. While the rapid $t^{-5 / 3}$ decline of this fallback rate [1] implies that $1 \%$ of $\dot{M}_{\text {Edd }}$ is typically reached within a few to ten years. A tidal disruption flare (TDF) may thus be used to sample different modes of accretion for a single supermassive black hole, and thus test our understanding of accretion physics.

Tens of (candidate) stellar tidal disruption events have been found by searching for flares in soft X-ray (e.g., [2-6]), UV [7-9], or optical surveys [10-12], or based on spectra with extreme coronal lines $[13,14]$. None of these thermal flares are associated with a radio transient, but only a handful have been followed up at this frequency. The only tidal disruption candidates with a detected transient radio counterpart are those discovered in $\gamma$-rays by Swift: Sw 1644+57 [15-18] and Sw 2058+05 [19]. Since the radio and X-ray emission of these two events most likely originates from a relativistic jetted outflow, they are often referred to as relativistic TDFs. In this paper we shall refer to the other class of TDFs as 'thermal', since they are all discovered at optical to soft X-ray frequencies.

One is left to wonder why the two TDFs discovered with Swift are the only events with evidence for a newly-born jet. Interpreting this as a radio-loud/radio-quiet dichotomy, similar to the devision of radio-loudness in quasars [20,21], would require that the tidal disruption jet launching mechanism is sensitive to the properties of the

\footnotetext{
a e-mail: s.vanvelzen@astro.ru.nl
}

disruption (e.g., mass ratio, impact parameter, orientation of the orbit of the star with respect to the black hole spin, or circumnuclear environment). On the other hand, based on the observation of a fundamental plane of black hole accretion, a universal scaling law for the non-thermal emission across the entire black hole mass range [22,23], one may postulate that all stellar tidal disruptions launch jets.

This proceeding is a condensed version of a forthcoming publication (van Velzen, Frail, Körding, and Falcke 2012, submitted). In sec. 2 we present two different tidal disruption (TD) jet models and compute off-axis light curves. In sec. 3 we discuss the radio observations and sample selection. We use these observations to constrain the jet models in sec. 4 and we close with a discussion in sec. 5 .

\section{TIDAL DISRUPTION JET MODELS}

In this section we review two models of tidal disruption jets and we present off-axis light curves for these models. We have divided the models into two classes based on the origin of the emitting particles: external or internal. In both models, some fraction of the accretion power ends up in the jet and the emission mechanism is synchrotron radiation.

\subsection{External model: Off-axis light curves for Sw 1644+57}

In the external model of radio emission from TD jets $[25,26]$, shock interaction between the jet and the gaseous circumnuclear medium powers the emission, similar to 


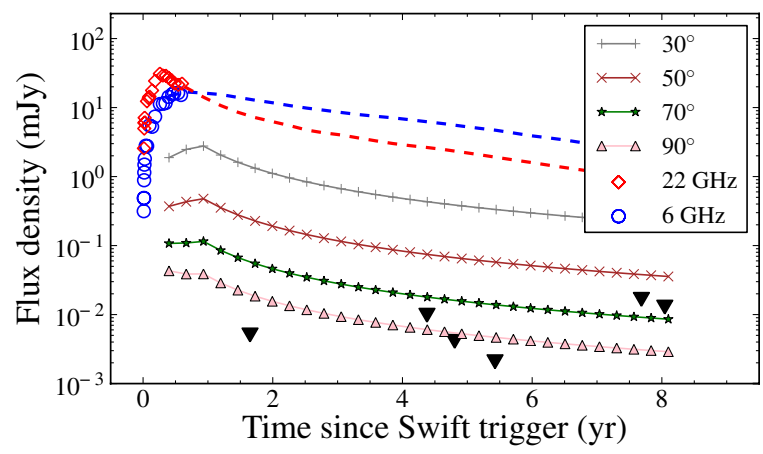

Figure 1. The observed light curve of $\mathrm{Sw} 1644+57$ (open symbols), with the predicted late-time light curve (dashed lines) for a total jet energy of $E_{j}=10^{52}$ erg [24]. We show the estimated $5 \mathrm{GHz}$ light curve of different off-axis observers, Eq. 2, assuming that the Lorentz factor of the jet decreases with $\Gamma_{j} \propto$ $t^{-0.2}$. We modified the extrapolated light curve to match a SedovTaylor solution, $L_{j} \propto t^{-1.2}$, when $\Gamma_{j}<2$. The 2- $\sigma$ upper limits on the radio flux of seven other TDF candidates (Table 1) are shown with black triangles (we scaled these limits to the redshift of Sw 1644+57, see sec. 4).

afterglow models of gamma-ray bursts. The model has been applied to the radio light curve of the relativistic TDF Sw 1644+57 [24,26], we show the fit and predicted latetime light curve in Fig. 1. We note that this fit requires an continuous increase of the isotropic jet power during the first year of observations.

The scaling of the synchrotron peak and self absorption frequency external model of $\mathrm{Sw} 1644+57$ are based on spherical expansion of an ultra-relativistic shell and thus require $\theta_{j} \Gamma_{j}<1$ (with $\theta_{j}, \Gamma_{j}$ the jet opening angle and Lorentz factor, respectively), plus an on-axis observer $i_{1}<1 / \Gamma_{j}(t=0)$; both requirements are supported by the observed radio light curve [26].

To compute the light curve for an off-axis observer, we first boost the observed on-axis flux $F_{1}(v)$ into the jet restframe

$$
L_{j}(v)=d_{L}^{2} \delta_{1}^{3-\alpha} F_{1}(v)
$$

(e.g., $[27,28])$ here we introduced the Doppler factor for the on-axis observer $\delta_{1}=\left[\Gamma_{j}\left(1-\beta_{j} \cos i_{1}\right)\right]^{-1}$ with $\beta_{j}=$ $v_{j} / c, \alpha$ is the spectral index defined as $F(v) \propto v^{\alpha}$, and $d_{L}$ is the luminosity distance. Next, we transform the jet luminosity to the off-axis observer using a different Doppler factor, $\delta_{2}$. If the size of the emitting region is small compared to the distance to the black hole, the time delay due to the geometrical separation of the synchrotron peak with frequency can be ignored, and we can estimate the flux for an observer sitting at $i_{2}$ :

$$
\begin{aligned}
F_{2}(t, v) & =\left(\frac{\delta_{2}}{\delta_{1}}\right)^{3-\alpha(t)} F_{1}(t, v) \\
& \approx\left(\frac{1-\beta_{j}(t)}{1-\beta_{j}(t) \cos i_{2}}\right)^{3-\alpha(t)} F_{1}(t, v) .
\end{aligned}
$$

Here $t$ is measured in the observer-frame, $\alpha(t)$ is obtained from the light curve, and we used $\cos i_{1} \approx 1-\Gamma_{j}^{-2} / 2 \sim$ 1 to simplify the equation. If $\mathrm{Sw} 1644+57$ was indeed a relativistic outflow that we observed on-axis, the light curve for off-axis observers depends only $\Gamma_{j}(t)$ and $i_{2}$. The latter is a free-parameter, which we shall constrain by our follow-up observations (in sec. 4).

The external model of TD jets [26], applied to Sw $1644+57$ [24], is used to obtain $\Gamma_{j}(t)$ and the light curve beyond the last published radio observation of Sw 1644+57 (about one year after the Swift trigger). The off-axis light curve that is derived here may thus be viewed as a test for this model. We consider two scenarios. First, we set $\Gamma(t>1 \mathrm{yr})=2$, and use the extrapolated light curve presented in [24]. This constant Lorentz factor is required because the extrapolated light curve is no longer valid when the jet slows down to mildly relativistic speed and lateral expansion becomes important. This happens at $\Gamma_{j} \lesssim 2$ [29]. We also consider a decreasing jet velocity $\Gamma_{j} \propto t^{-0.2}$ (as inferred for Sw $1644+57$ ), but modify the extrapolated light curve to match the non-relativistic Sedov-Taylor evolution, $L_{j} \propto t^{-1.2}$, when $\Gamma_{j}<2$. We show the light curves in Fig. 1.

\subsection{Internal model: Off-axis light curves for known TDFs}

The internal model of radio emission from TD [30,31], is based on the simple idea of jet-disk coupling [32,33]: a constant fraction of the accretion luminosity $\left(L_{d}\right)$ is fed into the jet, $Q_{j}=q_{j} L_{d}$. The conversion from jet power $\left(Q_{j}\right)$ to radio luminosity $\left(L_{j}\right)$ follows by assuming equipartition between the energy in relativistic particles and magnetic fields, and has been calibrated using observations of AGN [34-36].

Stellar mass black holes show rapid switches from radio-loud $\left(q_{j}=0.2\right)$ to radio-quiet $\left(q_{j}<0.002\right)$ coupling as the accretion rate increases from sub-Eddington to (near) the Eddington limit [37]. Motivated by the growing evidence that accretion onto super-massive black holes can also divided into these two modes (e.g.,[38-40]), we considered the following three scenarios for the jet-disk coupling in tidal disruptions:

$$
q_{j}=\left\{\begin{array}{llr}
0.2 & \text { all times } & (a) \\
0.002 & \dot{M}(t)>2 \% \dot{M}_{\text {Edd }}(b) \\
0.2 & t<t_{\text {fallback }}(c)
\end{array}\right.
$$

where each scenario reverts to the preceding one if the condition on $t$ or $\dot{M}$ is not true (e.g., $q_{j}=0.2$ when $\dot{M}<2 \% \dot{M}_{\text {Edd }}$ in all three scenarios). In the optimistic scenario $(a)$, the TD jet behaves like a radio-loud quasar at all times. In the most conservative scenario $(b)$, the jet becomes radio-loud only when the accretion drops below $<2 \% \dot{M}_{\text {Edd }}$. While in $c$, the system starts with a radioloud burst during the onset of the accretion. We consider $c$ the most realistic scenario, since it resembles most closely what is observed in X-ray binaries.

Besides $q_{j}$ (Eq. 3) the internal model requires a jet Lorentz factor and the disk luminosity as a function of time. The latter is obtained from the fallback rate of the stellar debris for a pericenter passage at the disruption radius, capped at the Eddington limit. We use $\Gamma_{j}=5$, the default value for this model [31]. 
Table 1. Jansky VLA observations at $5 \mathrm{GHz}$ of TDF candidates discovered at UV to optical frequencies. We list the redshift and estimated black hole mass of these candidates in the second and third column, respectively. No significant emission was detected at the phase center of the images. We list integration time after removal of interference, the rms of the images, and the time of the observations with respect to the estimated time of disruption.

\begin{tabular}{cccccc}
\hline name & redshift & $\begin{array}{c}M_{\mathrm{BH}} \\
M_{\odot} \times 10^{7}\end{array}$ & $\begin{array}{c}t_{\text {int }} \\
(\mathrm{min})\end{array}$ & $\begin{array}{c}\sigma\left(F_{v}\right) \\
(\mu \mathrm{Jy})\end{array}$ & $\begin{array}{c}\Delta t \\
(\mathrm{yr})\end{array}$ \\
\hline \hline D1-9 [7] & 0.326 & 5 & 30 & 9 & 8.0 \\
D3-13 [7] & 0.370 & 2 & 18 & 8 & 7.6 \\
TDE1 [10] & 0.136 & 1 & 28 & 10 & 5.4 \\
D23H-1 [8] & 0.186 & 5 & 28 & 8 & 4.8 \\
TDE2 [10] & 0.252 & 5 & 25 & 12 & 4.3 \\
PTF10iya [12] & 0.224 & 1 & 18 & 8 & 1.6 \\
\hline
\end{tabular}

\section{OBSERVATIONS}

To increase the number of TDF with radio follow-up observations, we selected all TDF candidates with an estimated time of disruption after 2004 for follow-up observations. This limit is used since the internal model of TD jets is no longer valid when the jet slows down significantly. Our sample also includes TDFs with existing radio upper limits, since the radio emission can be delayed with respect to the time of disruption, see previous section. We removed CSS100217 from the sample because it is detected at $1 \mathrm{GHz}$ before the time of disruption with a flat spectral index, indicating an AGN origin for the radio emission [11]. SDSS J1201+30 was not selected for follow-up observations because this TDF was published after our observations were scheduled. Details of the data reduction of the remaining six candidates are summarized below.

The radio observations were carried out on the Karl G. Jansky Very Large Array in the C configuration on 29 January 2012 under program 12A-005. We observed at a central frequency of $5.0 \mathrm{GHz}$ with 16 subbands each with $642 \mathrm{MHz}$ channels, spanning $2 \mathrm{GHz}$ of total bandwidth. We summarize the results of these observations in Table 1. No significant emission was detected at the phase center of the images.

\section{ANALYSIS}

In this section we compute the constraints that can be placed on TD jet models using our Jansky VLA follow-up observation.

If we assume that the angle between the observer and the jet is drawn from a uniform distribution (on a sphere), we can calculate the probability of non-detections for a given flux density limit. One simply has to find the largest angle for which the predicted flux is above the flux limit and then calculate the probability to observe a jet within this angle. The flux limit is set at twice the rms of the radio image of each TDF. (This is lower than the limit for a blinddetection experiment since we use the threshold to find the probability of a non-detection, not to claim a discovery.) In Table 2 we list the results of this exercise.
Table 2. Probability (\%) that the jet orientation is such that the predicted flux is below the $2 \sigma$-level of the $5 \mathrm{GHz}$ observation. Zero probability implies the predicted flux is above the threshold even for $i_{2}=\pi / 2$, while $P_{i}=100 \%$ implies the data cannot constrain the model. In the second to third column we list the results for the internal jet model, for the optimistic to the conservative scenario (Eq. 3), using $\Gamma_{j}=5$. In the fourth and fifth column we give the probably of detecting a jet that is identical to Sw $1644+57$, but observed off-axis, using two different estimates of the light curve past the last available observation (see sec. 2.1).

\begin{tabular}{cccccc}
\hline name & \multicolumn{3}{c}{ Internal jet model } & \multicolumn{2}{c}{ Sw 1644+57, off-axis } \\
& $a$ & $c$ & $b$ & $\Gamma_{j}=2$ & $\Gamma_{j} \propto t^{-0.2}$ \\
\hline \hline D1-9 & 39 & 78 & 83 & 49 & 46 \\
D3-13 & 62 & 89 & 91 & 52 & 50 \\
TDE1 & 7 & 92 & 100 & 0 & 0 \\
D23H-1 & 0 & 52 & 70 & 0 & 0 \\
TDE2 & 0 & 75 & 98 & 20 & 19 \\
PTF10iya & 0 & 86 & 95 & 0 & 0 \\
\hline
\end{tabular}

The probability that all six TDFs in our sample hosted jets, but were not detected due to Doppler boosting is $P_{6}=$ $\Pi_{i} P_{i}$, with $P_{i}$ being the probability of the observations of each TDF candidate, as listed in Table 2. For the optimistic scenario of the internal model (sec. 2.2) with $\Gamma_{j}=5$, three of the six TDF candidates (D23H-1, TDE2, PTF10iya) should have yielded a detection above the 2- $\sigma$ level, hence $P_{6}=0$. The probability that all of the other three TDF candidates hosted a jet is $1.7 \%$. For the most conservative scenario (Eq. $3 b), P_{6}=49 \%$, while for the realistic scenario (Eq. $3 c$ ) this is lower at $21 \%$.

Our upper limits also constrain the possibility that a jet similar to $\mathrm{Sw} 1644+57$ was launched after the disruption. To place the Jansky VLA observations on the estimated off-axis light curve (Eq. 2), we equate the time of disruption to the time of the Swift trigger and we scale the flux using $\left(d_{L, \mathrm{Sw}} / d_{L}\right)^{2}$, with $d_{L, \mathrm{Sw}}$ being the luminosity distance of $\mathrm{Sw} 1644+57$. From Fig. 1 we see that our upper limits on the radio flux of three TDFs (TDE1, D23H-1, PTF10iya) are inconsistent with the estimated off-axis light curve of $\mathrm{Sw} 1644+57$ for all viewing angles. The probability that the other three flares hosted a radio transient identical to $\mathrm{Sw} 1644+57$ is about $5 \%$ (for both versions of the late-time evolution we considered in sec. 2.1).

\section{CONCLUSION \& DISCUSSION}

We obtained upper limits at the $\sim 10 \mu \mathrm{Jy}$ level of the $5 \mathrm{GHz}$ flux of six stellar tidal disruptions events that were discovered with optical/UV imaging surveys. This is three orders of magnitude lower than the recently discovered TDFs with radio emission. Suggesting that stellar tidal disruptions come in different flavors, ranging from radio-loud to radio-quiet (or radio-silent). To explore how this conclusion would be biased by the large possibile parameter range inherent to TDFs, we compared our upper limits to currently available jet models, taking into account Doppler boosting and temporal evolution of the radio emission. 
We used our observations to constrain the internal jet model (sec. 2.2). For a jet Lorentz factor of $\Gamma_{j}=5$, we can rule out the optimistic ("alway radio-loud") scenario for three of the six flares. The probability that the other three TDF candidates did launch such jets, but are not detected because Doppler boosting reduced the flux below two times the image rms is only $4 \%$. The hypothesis that all events hosted jets that only becomes radio-loud when the fallback rate drops below $2 \%$ of the Eddington accretion rate (i.e., as observed in stellar mass black holes) is less constrained.

We have also investigated the possibility that our sample of TDFs hosted a jet which is identical to Sw 1644+57, but oriented at a larger angle between the observer and the jet. For three of the six flares, the estimated off-axis light curves of this relativistic TDF are inconsistent with the non-detection for all possible observer angles. The hypothesis that all of the other TDFs hosted jets identical to Sw $1644+57$ is ruled out at the $95 \%$ confidence level. A caveat is that the off-axis light curves we used in this work are only valid for circumnuclear environments that are identical to the host of $\mathrm{Sw} 1644+57$.

If a division between tidal disruptions with and without jets indeed exists, it presents a challenge to the idea that radio-loudness can be explained by state changes of accretion disk. Some authors have argued that the spin of the black hole is important to produce stellar tidal disruption jets (e.g., [41-43]). Testing this idea requires observations of the disk emission of tidal disruptions with jets to show that this component is similar to TDFs without jets. Otherwise, the observed range in radio-loudness can also be explained by a different evolution of the stellar debris.

\section{References}

[1] M.J. Rees, Nature 333, 523 (1988)

[2] S. Komossa, N. Bade, A\&A 343, 775 (1999)

[3] D. Grupe, H.C. Thomas, K.M. Leighly, A\&A 350, L31 (1999)

[4] P. Esquej, R.D. Saxton, S. Komossa, A.M. Read, M.J. Freyberg, G. Hasinger, D.A. García-Hernández, H. Lu, J.R. Zaurín, M. Sánchez-Portal et al., A\&A 489, 543 (2008)

[5] W.P. Maksym, M.P. Ulmer, M. Eracleous, ApJ 722, 1035 (2010)

[6] R.D. Saxton, A.M. Read, P. Esquej, S. Komossa, S. Dougherty, P. Rodriguez-Pascual, D. Barrado, A\&A 541, A106 (2012)

[7] S. Gezari, S. Basa, D.C. Martin, G. Bazin, K. Forster, B. Milliard, J.P. Halpern, P.G. Friedman, P. Morrissey, S.G. Neff et al., ApJ 676, 944 (2008)

[8] S. Gezari, T. Heckman, S.B. Cenko, M. Eracleous, K. Forster, T.S. Gonçalves, D.C. Martin, P. Morrissey, S.G. Neff, M. Seibert et al., ApJ 698, 1367 (2009)

[9] S. Gezari, R. Chornock, A. Rest, M.E. Huber, K. Forster, E. Berger, P.J. Challis, J.D. Neill, D.C. Martin, T. Heckman et al., Nature 485, 217 (2012)
[10] S. van Velzen, G.R. Farrar, S. Gezari, N. Morrell, D. Zaritsky, L. Östman, M. Smith, J. Gelfand, A.J. Drake, ApJ 741, 73 (2011)

[11] A.J. Drake, S.G. Djorgovski, A. Mahabal, J. Anderson, R. Roy, V. Mohan, S. Ravindranath, D. Frail, S. Gezari, J.D. Neill et al., ApJ 735, 106 (2011)

[12] S.B. Cenko, J.S. Bloom, S.R. Kulkarni, L.E. Strubbe, A.A. Miller, N.R. Butler, R.M. Quimby, A. Gal-Yam, E.O. Ofek, E. Quataert et al., MNRAS 420, 2684 (2012)

[13] S. Komossa, H. Zhou, T. Wang, M. Ajello, J. Ge, J. Greiner, H. Lu, M. Salvato, R. Saxton, H. Shan et al., ApJ 678, L13 (2008)

[14] T.G. Wang, H.Y. Zhou, S. Komossa, H.Y. Wang, W. Yuan, C. Yang, ApJ 749, 115 (2012)

[15] J.S. Bloom, D. Giannios, B.D. Metzger, S.B. Cenko, D.A. Perley, N.R. Butler, N.R. Tanvir, A.J. Levan, P.T. O'Brien, L.E. Strubbe et al., Science 333, 203 (2011)

[16] D.N. Burrows, J.A. Kennea, G. Ghisellini, V. Mangano, B. Zhang, K.L. Page, M. Eracleous, P. Romano, T. Sakamoto, A.D. Falcone et al., Nature 476, 421 (2011)

[17] A.J. Levan, N.R. Tanvir, S.B. Cenko, D.A. Perley, K. Wiersema, J.S. Bloom, A.S. Fruchter, A.d.U. Postigo, P.T. O'Brien, N. Butler et al., Science 333, 199 (2011)

[18] B.A. Zauderer, E. Berger, A.M. Soderberg, A. Loeb, R. Narayan, D.A. Frail, G.R. Petitpas, A. Brunthaler, R. Chornock, J.M. Carpenter et al., Nature 476, 425 (2011)

[19] S.B. Cenko, H.A. Krimm, A. Horesh, A. Rau, D.A. Frail, J.A. Kennea, A.J. Levan, S.T. Holland, N.R. Butler, R.M. Quimby et al., ApJ 753, 77 (2012)

[20] K.I. Kellermann, R. Sramek, M. Schmidt, D.B. Shaffer, R. Green, AJ 98, 1195 (1989)

[21] H. Falcke, W. Sherwood, A.R. Patnaik, ApJ 471, 106 (1996)

[22] A. Merloni, S. Heinz, T. di Matteo, MNRAS 345, 1057 (2003)

[23] H. Falcke, E. Körding, S. Markoff, A\&A 414, 895 (2004)

[24] E. Berger, A. Zauderer, G.G. Pooley, A.M. Soderberg, R. Sari, A. Brunthaler, M.F. Bietenholz, ApJ 748, 36 (2012)

[25] D. Giannios, B.D. Metzger, MNRAS 416, 2102 (2011)

[26] B.D. Metzger, D. Giannios, P. Mimica, MNRAS 420, 3528 (2012)

[27] K.R. Lind, R.D. Blandford, ApJ 295, 358 (1985)

[28] S. Jester, MNRAS 389, 1507 (2008)

[29] W. Zhang, A. MacFadyen, ApJ 698, 1261 (2009)

[30] S. van Velzen, H. Falcke, G.R. Farrar, in ISKAF2010 Science Meeting (2010)

[31] S. van Velzen, E. Körding, H. Falcke, MNRAS 417, L51 (2011)

[32] S. Rawlings, R. Saunders, Nature 349, 138 (1991)

[33] H. Falcke, P.L. Biermann, A\&A 293, 665 (1995)

[34] H. Falcke, M.A. Malkan, P.L. Biermann, A\&A 298, 375 (1995) 
[35] C.J. Willott, S. Rawlings, K.M. Blundell, M. Lacy, MNRAS 309, 1017 (1999)

[36] E.G. Körding, S. Jester, R. Fender, MNRAS 383, 277 (2008)

[37] R.P. Fender, T.M. Belloni, E. Gallo, MNRAS 355, 1105 (2004)

[38] L.C. Ho, ApJ 516, 672 (1999)
[39] E.G. Körding, S. Jester, R. Fender, MNRAS 372, 1366 (2006)

[40] P.N. Best, T.M. Heckman, MNRAS 421, 1569 (2012)

[41] W.H. Lei, B. Zhang, ApJ 740, L27 (2011)

[42] J.H. Krolik, T. Piran, ArXiv e-prints (2011)

[43] N. Stone, A. Loeb, Physical Review Letters 108, 061302 (2012) 